\title{
Experimental Comparison of UTC- and PIN-Photodiodes for Continuous-Wave Terahertz Generation
}

\author{
S. Nellen ${ }^{1}$ (D) - T. Ishibashi ${ }^{2} \cdot$ A. Deninger ${ }^{3} \cdot$ R. B. Kohlhaas ${ }^{1} \cdot$ L. Liebermeister $^{1}$ $^{\circ}$ \\ M. Schell ${ }^{1} \cdot$ B. Globisch ${ }^{1}$
}

Received: 12 June 2019 / Accepted: 21 October 2019 /Published online: 20 December 2019

(C) The Author(s) 2019

\begin{abstract}
We carried out an experimental comparison study of the two most established optoelectronic emitters for continuous-wave $(\mathrm{cw})$ terahertz generation: a uni-traveling-carrier photodiode (UTC-PD) and a pin-photodiode (PIN-PD). Both diodes are commercially available and feature a similar package (fiber-pigtailed housings with a hyper-hemispherical silicon lens). We measured the terahertz output as a function of optical illumination power and bias voltage from $50 \mathrm{GHz}$ up to $1 \mathrm{THz}$, using a precisely calibrated terahertz power detector. We found that both emitters were comparable in their spectral power under the operating conditions specified by the manufacturers. While the PIN-PD turned out to be more robust against varying operating parameters, the UTC-PD showed no saturation of the emitted terahertz power even for $50 \mathrm{~mW}$ optical input power. In addition, we compared the terahertz transmission and infrared (IR) blocking ratio of four different filter materials. These filters are a prerequisite for correct measurements of the absolute terahertz power with thermal detectors.
\end{abstract}

Keywords Terahertz source $\cdot$ Continuous-wave terahertz (cw THz) $\cdot$ Millimeter-wave $\cdot$ Optoelectronics $\cdot$ Photonics $\cdot$ Microwave photonics $\cdot$ Photodiode $\cdot$ Uni-traveling-carrier photodiode (UTC-PD) $\cdot$ P-i-n photodiode (PIN-PD)

\section{Introduction}

Within the last decade, terahertz technologies attracted more and more interest due to an evergrowing number of industrial, "real-world" applications. Originally invented for scientific

S. Nellen

simon.nellen@hhi.fraunhofer.de

1 Fraunhofer Institute for Telecommunications, Heinrich Hertz Institute, Einsteinufer 37, 10587 Berlin, Germany

2 NTT Electronics Techno Corporation, Atsugi 243-0198, Japan

3 TOPTICA Photonics AG, Lochhamer Schlag 19, 82166 Gräfelfing, Germany 
research, terahertz techniques have found their way into non-destructive testing [1], process control [2, 3], imaging [4], composition analysis [5-7], and wireless communications at carrier frequencies up to $600 \mathrm{GHz}[8,9]$. Among the multitude of techniques for generating and detecting terahertz radiation, optoelectronic, continuous-wave $(\mathrm{cw})$ terahertz devices combine broad frequency tuning [10, 11] with high accuracy and sensitivity [12]. Furthermore, choosing an optical excitation wavelength in the c-band (1530 to $1565 \mathrm{~nm}$ ) enables the use of mature and reliable components developed for fiber-optical communications, and, therefore, the design and build of compact and cost-efficient cw terahertz systems [13, 14].

For $\mathrm{cw}$ terahertz emission, a photodiode can efficiently convert an optical signal into an electrical one by so-callled "photomixing." To this end, a pair of single-mode lasers generates an optical beat with frequency $f_{\text {beat }}=\left|f_{\text {Laser1 }}-f_{\text {Laser } 2}\right|$. For instance, choosing two lasers with emission wavelengths of $1540 \mathrm{~nm}(194.67 \mathrm{THz})$ and $1547.95 \mathrm{~nm}(193.67 \mathrm{THz})$, respectively, results in an optical beat with $f_{\text {beat }}=1 \mathrm{THz}$. When fed into a photodiode, the two-color signal induces a photocurrent that is modulated at the beat frequency. An antenna attached to the diode radiates the terahertz signal into free space. When tunable lasers are used as optical sources, the difference frequency of the beat note can be changed over a broad spectral range, which translates directly into widely tunable terahertz emission.

Figure 1 shows schematic band diagrams of the two different types of photodiodes, which are commonly used today: the PIN-PD and the UTC-PD. The PIN-PD is based on an intrinsic (i) absorption layer sandwiched between positively (p-type) and negatively (n-type) doped layers. Photon absorption generates electron-hole-pairs in the depleted i-layer. Driven by the intrinsic electric field and an external bias voltage, photogenerated electrons and holes travel into the $\mathrm{n}$ - and p-layer, respectively. Thus, both types of carriers contribute to the photocurrent in the device. While the comparably low effective mass of the electrons enables a fast acceleration of these carriers, the holes are 10 to 20 times slower due to their higher effective mass in III-V semiconductors [15]. This high effective mass of the holes ultimately leads to a saturation of the high-frequency photocurrent in PIN-PDs, especially for high optical excitation densities. Thus, the transit time of holes traveling from the intrinsic layer into the P-layer becomes the main factor that determines the cutoff frequency of PIN-PDs.

In order to overcome this transit time limitation of PIN-PDs, UTC-PDs have been developed in the late 1990s [16]. UTC diodes rely on a p-type absorption layer next to an intrinsic carrier collection layer (see Fig. 1b). This modified layer structure spatially separates photon absorption and carrier acceleration. Since the p-doped absorber is close to the p-layer, holes do not need to cross the carrier collection layer, but reach the p-layer within a very short traveling time. Only the fast electrons are injected into the depleted carrier collection layer, where they are accelerated by the intrinsic electric field of the collection layer to reach the n-layer. In order to avoid electron diffusion into the p-contact, a blocking layer serves as barrier in the conduction band $(\mathrm{CB})$ between p-layer and p-absorber. At the same time, hole diffusion into the carrier collection layer is suppressed by the valence band (VB) cliff, i.e., a barrier in the VB between absorber and collection layer [17, 18].

Both PIN-PDs and UTC-PDs were originally developed as detectors in fiber-optical communication networks $[16,19]$. Later on, they were modified to match the requirements for cw terahertz emission [20-26]. A detailed review on the technologies and, in particular, the differences between PIN- and UTC-PDs can be found in reference [27].

In this paper, we compare commercially available PIN- and UTC-emitters with respect to their frequency-dependent absolute terahertz output power as a function of the optical input power and the external bias voltage. We relate the observed differences in frequency response 
a)

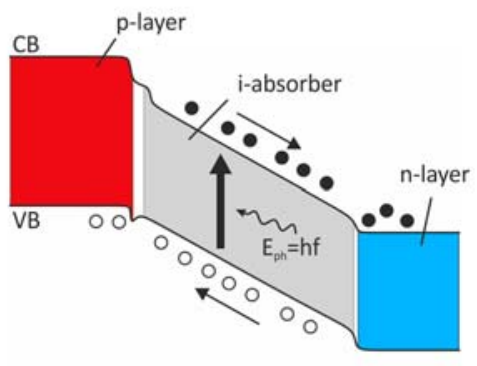

b)

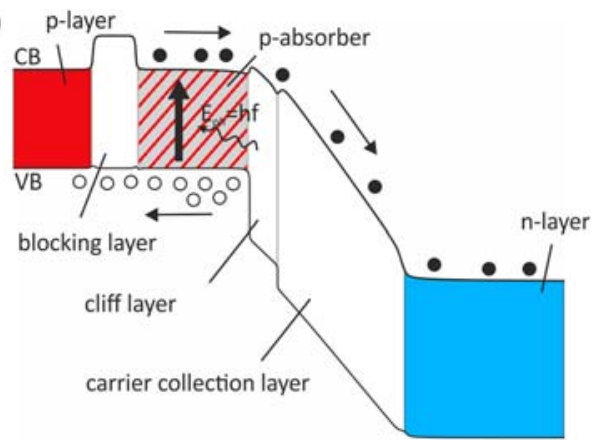

Fig. 1 Schematic band diagram of a PIN-PD (a) and a UTC-PC (b). a Incoming light with energy $\mathrm{E}_{\text {ph }}$ generates free electrons in the conduction band (CB) and holes in the valence band (VB) of the intrinsic absorber region (grey). Due to the intrinsic electric field, electrons move to the n-layer (blue) and holes to the p-layer (red). Grading layers (white) between $\mathrm{p}$-, i-, and n-layer ensure smooth junctions. b Incoming light generates free electrons in the CB and holes in the VB of the p-type absorber (grey/red) of the UTC-PD. The blocking layer between p-layer (red) and absorber prevents electrons from diffusing into the p-layer. Thus, only electrons are injected into the carrier collection layer to reach the n-layer (blue). Photogenerated holes can reach the p-layer without any blocking in the VB. However, the cliff layer in the VB introduces a barrier that blocks the diffusion of holes into the collection layer

and saturation behavior directly to the particular type of diode and terahertz antenna attached to it. Before presenting the results of this comparison, we describe the experimental setup, including a detailed comparison of four different absorber materials that are transparent for terahertz radiation but block infrared light (IR). These IR attenuators are mandatory to perform absolute terahertz power measurements with thermal terahertz detectors. To the best of our knowledge, this is the first comparison study not only of PIN- and UTC-PDs but also of these IR absorbers.

\section{Experimental Setup}

For the terahertz power measurements, we employed a pyroelectric thin-film (PTF) detector (model THz20 from Sensor- und Lasertechnik $\mathrm{GmbH}$ ), which is calibrated by the German National Metrology Institute (PTB) [28, 29]. Figure 2a shows a sketch of the setup as used for both the PIN- and UTC-emitters. Two external-cavity lasers (ECLs, model 81960A and $81608 \mathrm{~A}$ from Keysight) emit light at wavelengths $\lambda_{1}=1540 \mathrm{~nm}$ and $\lambda_{2}=1540 \mathrm{~nm}+f_{\mathrm{THz}}$, respectively. Combining the two ECL outputs produces an optical beat with envelope frequency $f_{\mathrm{THz}}$. A semiconductor optical amplifier (SOA, model BOA1004P from Thorlabs) boosts the optical beat to the desired power level to drive the photodiode. The frequency of the terahertz radiation $f_{\mathrm{THz}}$ is changed by tuning the wavelength $\lambda_{2}$ of the second ECL. The PTF detector is placed at a distance of $10 \mathrm{~mm}$ from the terahertz emitter. The circular sensor area of the PTF detector has a diameter of $20 \mathrm{~mm}$, which is sufficient to collect the emitted terahertz signal, and therefore no collimating or focusing terahertz optics are needed (see Fig. 2b). A waveform generator (model 3390 from Keithley) produces a $20 \mathrm{~Hz}$ square wave that modulates the bias voltage of the photodiode between $0 \mathrm{~V}$ and the chosen operating voltage. The modulation enables the use of a lock-in amplifier (LIA, model 7265 from Signal Recovery). Before the LIA, a transimpedance amplifier (TIA, CurrentPreAmplifier from 
Sensor- und Lasertechnik) is employed. A multimeter (model 22105 1/2 from Keithley) measures the DC current of the photodiode.

Due to an impedance mismatch between waveform generator and terahertz emitter, the terminal voltage of the waveform generator is not the actual voltage applied to the terahertz emitter. While the output termination of the waveform generator is $R_{i}=50 \Omega$, the resistance of the illuminated photodiode changes with the optical illumination power. In order to correct this mismatch, a multimeter measures the photocurrent of the diode, and the actual voltage $V_{\mathrm{db}}$ at the biasing port of the device is determined via $V_{\mathrm{db}}=V_{i}-\left(R_{i}+R_{c}\right) \cdot I_{\mathrm{db}}$. Here, $V_{i}$ is the voltage applied by the waveform generator, $R_{i}$ is the output termination, $R_{c}$ is the resistance of the circuit, which consists of the multimeter and all electrical cables, and $I_{d b}$ is the photocurrent measured by the multimeter. Hence, $V_{\mathrm{db}}$ remains an accurate measure of the voltage applied at the biasing port of the emitter even if the resistance of the diode varies. In case of the PIN-PD emitter, the voltage $V_{\mathrm{PIN}}$ applied at the input connector of the PD is equal to the biasing voltage, hence $V_{\mathrm{PIN}}=V_{\mathrm{db}}$. In contrast, the UTC-PD is connected in parallel to a biasing resistor that reduces the voltage applied to the UTC-PD according to $V_{\mathrm{UTC}}=V_{\mathrm{db}}-\left(100 \Omega \cdot I_{\mathrm{db}}\right)$. In the remainder of this paper, bias voltages always refer to the corrected voltage $V_{\mathrm{db}}$ at the biasing port of the emitter.

Pyroelectric detectors are sensitive to both IR and terahertz radiation. In our setup, the lockin modulation is purely electronic. Thus, the lock-in amplifier eliminates any DC response of the PTF detector that might result from residual IR light. However, residual IR will still generate an offset in the output signal of the detector, and thus distort the terahertz measurement. For accurate terahertz power measurements, it is therefore essential to prevent any residual IR light from reaching the detector. For this purpose, commercially available PIN-PD emitters feature a black coating on the silicon lens, which reduces the residual IR at the terahertz output to less than $0.05 \%$ of the optical power fed into the PIN-PD. For comparison with the UTC-PD emitter, however, we removed this coating, such that both emitters featured an uncoated silicon lens. In order to quantify the residual IR, we replaced the PTF detector with an IR power detector (Thorlabs PM100D/S122C). The residual IR of the UTC and PIN module at $30 \mathrm{~mW}$ optical excitation power amounts to $4.02 \mathrm{~mW}(13.4 \%)$ and $2.88 \mathrm{~mW}$ $(9.6 \%)$, respectively. In our setup, the $10-\mathrm{mm}$ gap between emitter and pyroelectric detector allowed us to insert and characterize different materials with respect both to their IR absorption and terahertz transmission properties.

Employing the PIN-PD emitter, we examined four different IR absorbers, which are common materials used in experimental terahertz setups: (i) a $9 \mathrm{~mm}$ thick plate of foamed polystyrene, also known as Styropor ${ }^{\mathrm{TM}}$, (ii) an approx. $20 \mu \mathrm{m}$ thick black foil of HDPE, (iii) a $0.65 \mathrm{~mm} \mathrm{Al} \mathrm{O}_{3}$ ceramic plate with a black coating, and (iv) a $0.35 \mathrm{~mm}$ thick piece of black cardboard. Figure $2 \mathrm{~d}$ shows a photograph of absorber (i) inserted between emitter and terahertz detector. In the same way, we measured the IR transmission using the detector shown in Fig. 2c. In Fig. 2e, absorber (ii), the black HDPE foil, is placed in front of the emitter.

The plot on the left-hand side in Fig. 3 depicts the terahertz transmission of the four different materials at frequencies between $50 \mathrm{GHz}$ and $1 \mathrm{THz}$. For ease of comparison, we normalized the transmitted terahertz power to the power level without any filter. A low-pass Fourier filter removes oscillations in the transmission curves that stem from standing waves between the surfaces of the emitter, the detector, and the particular filter. Values higher than $100 \%$ result from the uncertainty of the PTF detector (6.9\%). Ceramic and cardboard appear transparent only at low frequencies $(<100 \mathrm{GHz})$ but exhibit a significant decrease in transmission towards higher frequencies. In contrast, foamed polystyrene and the HDPE foil show a comparatively flat transmission with an average value of more than $90 \%$ up to $1 \mathrm{THz}$. 

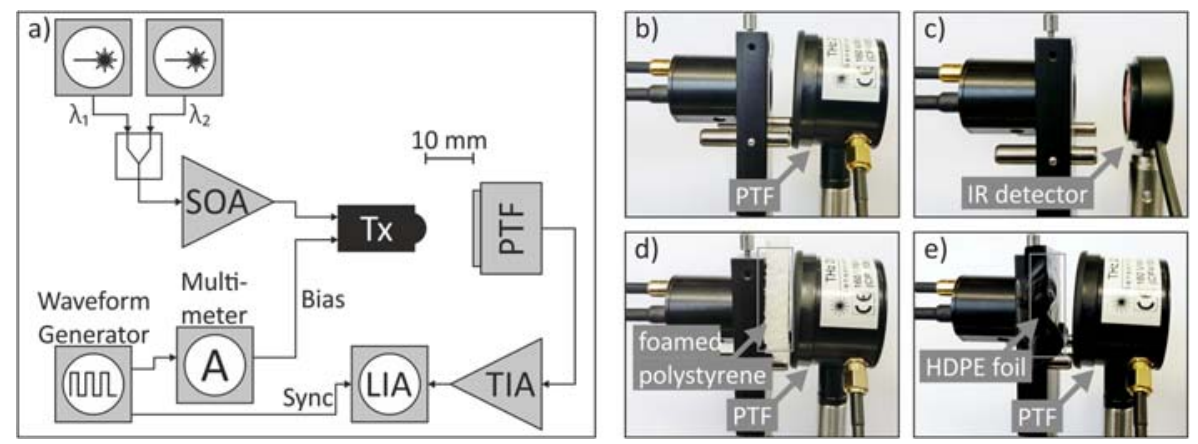

Fig. 2 a Schematic setup of the terahertz power measurement. Two external-cavity lasers $\left(\lambda_{1}, \lambda_{2}\right)$ are combined to generate an optical beat signal, which is amplified by a semiconductor optical amplifier (SOA) and fed into the photodiode emitter (Tx). A waveform generator modulates the bias voltage with a $20 \mathrm{~Hz}$ square wave between $0 \mathrm{~V}$ and the desired device bias $\mathrm{V}_{\mathrm{db}}$. A multimeter measures the resulting time-averaged DC photocurrent. The pyroelectric thin-film (PTF) detector is placed in front of the emitter at $10 \mathrm{~mm}$ distance. After amplification by a transimpedance amplifier (TIA), the detector signal is fed into a lock-in amplifier (LIA) referenced to the modulation frequency of the bias voltage (sync). b A photograph of the PIN-emitter in front of the detector. Note that no terahertz optics are used to guide the radiation from the emitter to the power detector ("face-to-face arrangement"). c The PTF detector is replaced by an infrared (IR) detector to determine the residual IR excitation from the emitter. In order to block the residual IR but transmit the terahertz signal, IR filters like foamed polystyrene (Styropor ${ }^{\mathrm{TM}}$ ) (d) or a black HDPE foil (e) can be inserted into the $10 \mathrm{~mm}$ gap between emitter and PTF detector

The bar chart on the right-hand side of Fig. 3 shows the measured IR absorption according to the setup shown in Fig. 2c. Black cardboard and ceramic block absorb almost $100 \%$ of the IR light, but as discussed above, this comes at a cost in terahertz transmittance, which drops to $50 \%$ for frequencies above $0.6 \mathrm{THz}$. On the opposite, the terahertz absorption of the HDPE foil

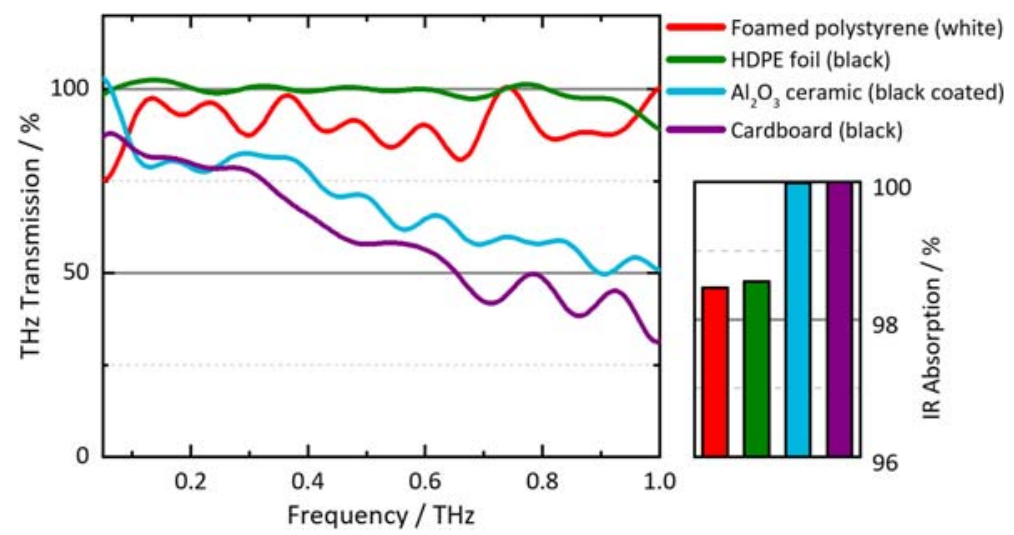

Fig. 3 Evaluation of potential filter materials for blocking residual IR light. Nine millimeters of foamed polystyrene (red curve), $20 \mu \mathrm{m}$ black HDPE foil (green curve), $0.65 \mathrm{~mm}$ ceramic with black coating (blue curve), and $0.35 \mathrm{~mm}$ thick black cardboard (purple curve). The $\mathrm{THz}$ power between $50 \mathrm{GHz}$ and $1 \mathrm{THz}$ is measured and normalized to the power spectrum without any filters in place. Foamed polystyrene and HDPE exhibit the lowest terahertz absorption. A low-pass Fourier filter reduces oscillations in the spectra that stem from standing waves between emitter, filter, and detector surface. The plot on the right-hand side shows the measured IR absorption. Note that all materials block more than $98 \%$ of the IR light, whereas their frequency-dependent terahertz transmission varies significantly 
and Styropor ${ }^{\mathrm{TM}}$ is negligibly low, and both materials show an IR absorption higher than 98.5\%. In the case of Styropor ${ }^{\mathrm{TM}}$, we observed a strong spatial dependence of the IR absorption, i.e., the IR absorption varied when the plate was moved perpendicular to the optical axis. We attribute this to the presence of millimeter-sized polystyrene grains, which change the optical path through the material depending on the illumination.

We therefore chose the HDPE foil as infrared absorber, due to its high terahertz transmission, fair IR blocking ratio, and ease in handling. Considering the amount of residual IR of the particular emitters, the fraction of the excitation power that reaches the PTF detector is only $0.20 \%$ in case of the UTC emitter and $0.14 \%$ in case of the PIN emitter. All of the terahertz measurements presented below were performed with the HDPE absorber in place.

\section{Results and Comparison}

In this paragraph, we present a detailed comparison of the emitted terahertz power as a function of frequency, optical excitation power, and bias voltage for the PIN-PD and the UTC-PD emitter.

Figure 4 shows the emitted power between $50 \mathrm{GHz}$ and $1 \mathrm{THz}$, at the recommended operating conditions given by the diode manufacturers, i.e., $30 \mathrm{~mW}$ optical power and $-1.5 \mathrm{~V}$ $(-1.0 \mathrm{~V})$ device bias for the PIN-PD (UTC-PD) emitter.

For frequencies between $400 \mathrm{GHz}$ and $1 \mathrm{THz}$ both emitters show a frequency roll-off of approx. $20 \mathrm{~dB}$ per decade, in agreement with the theoretical curve (dotted line in Fig. 4). We attribute this behavior to a first-order low pass arising from the $\mathrm{RC}$ constant of the photodiode. The RC bandwidth of an electromagnetic circuit is given by $f_{\mathrm{RC}}=\left(2 \pi R_{\mathrm{tot}} C_{\mathrm{PD}}\right)^{-1}$. Here, $f_{\mathrm{RC}}$ is the cutoff frequency, $C_{\mathrm{PD}}$ is the capacitance of the photodiode and $R_{\mathrm{tot}}$ is the total resistance with contributions from the diode $\left(R_{\mathrm{pd}}\right)$ and the antenna $\left(R_{\mathrm{ant}}\right)$ as shown in the simplified equivalent circuit of a PIN-PD in Fig. 5b. Note that in this equivalent circuit, the high resistance of the intrinsic absorber, which actually exists in parallel to the capacitance $C_{\mathrm{pd}}$, is neglected. This is a valid simplification because the capacitor $C_{\mathrm{pd}}$ is shorted for high frequencies [18]. Further, the impedance of the bow-tie antenna consists of a real part $\left(R_{\text {ant }}\right)$ only, since

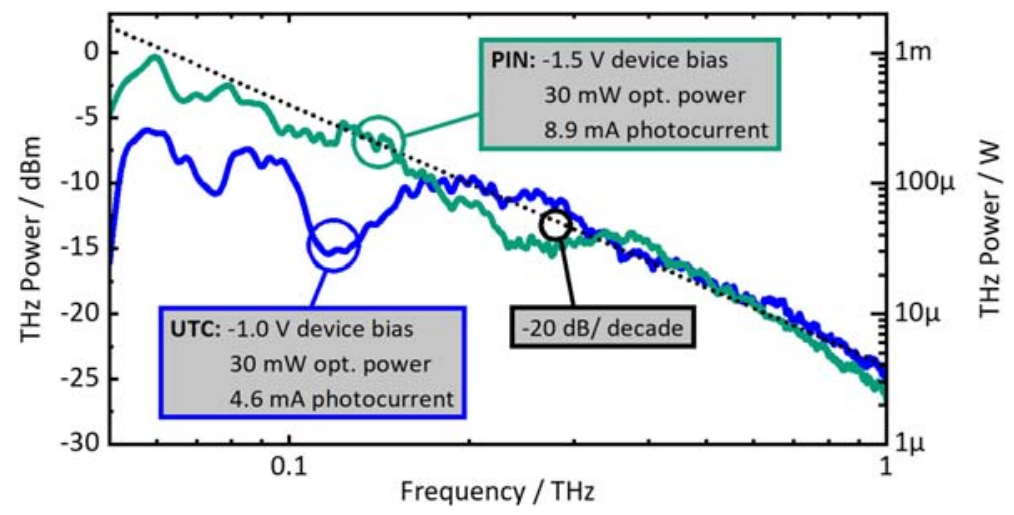

Fig. 4 Emitted terahertz power of PIN-PD (green curve) and UTC-PD (blue curve) emitters at their recommended operating conditions. $-1.5 \mathrm{~V}$ device bias and $30 \mathrm{~mW}$ optical power for the PIN-PD, and $-1.0 \mathrm{~V}$ device bias and $30 \mathrm{~mW}$ optical power for the UTC-PD. The dotted line indicates a decrease of $20 \mathrm{~dB}$ per decade corresponding to a first-order low pass 
a)

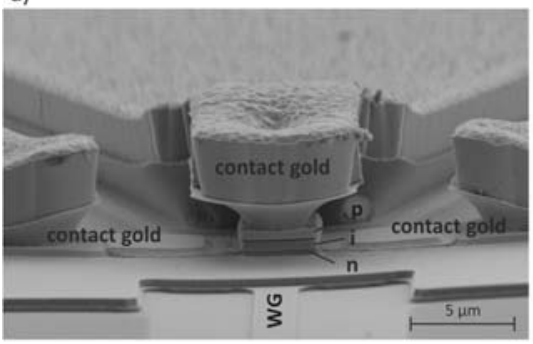

b)

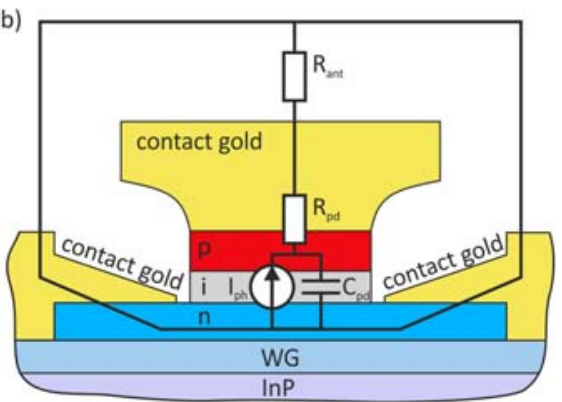

Fig. 5 a Scanning electron microscopy picture of a PIN-PD: p-, i-, and n-layer are stacked on top of the waveguide (WG) layer for optical excitation. The n-layer is connected to gold pads from the left and right side. An air bridge connects the p-layer to the gold pad in the background. $\mathbf{b}$ The simplified equivalent circuit consists of a capacitance $C_{p d}$ in parallel to the injection of the photocurrent $I_{p h}$. The photodiode resistance $R_{p d}$ and the antenna resistance $R_{a n t}$ are connected in series. Capacitance, resistance, and injected current correlate to the geometry of the PD and the material properties, illustrated by the cross-section overlay of the PIN-PD

the particular bow-tie antenna features a self-complementary structure and follows Babinet's principle [30, 31]. The fact that PIN-PD and UTC-PD show similar power levels for frequencies above $400 \mathrm{GHz}$ suggests that the 3-dB frequency of both devices is comparable. The resistance and the capacity of the PIN-PD amount to $65 \Omega$ and $19 \mathrm{fF}$, respectively, predicting a calculated RC-limit of $f_{\text {RC, PIN }} \approx 130 \mathrm{GHz}$, while $81 \Omega$ and $14 \mathrm{fF}$ of the UTC-PD predict $f_{\mathrm{RC} \text {, UTC }} \approx 140 \mathrm{GHz}$. Both values are in good agreement with the measured spectra shown in Fig. 4, i.e., starting to follow the $-20-\mathrm{dB}$ slope at the respective cutoff frequency.

Figure 4 further reveals significant differences between the two emitters at frequencies below $300 \mathrm{GHz}$. While the UTC-PD emits approx. $100 \mu \mathrm{W}$ at $250 \mathrm{GHz}$, the PIN-PD only produces $30 \mu \mathrm{W}$. In contrast, the PIN-PD emits $200 \mu \mathrm{W}$ at $130 \mathrm{GHz}$ compared with $30 \mu \mathrm{W}$ from the UTC-PD. We attribute this effect to the frequency-dependent impedance match between photodiode and antenna, which, in turn, results from different contact designs between photodiode and antenna (see Fig. 5a) as well as different antenna geometries. Even though both emitters feature a bow-tie antenna for broadband emission, differences in the antenna size and the geometry of the feeding point result in significant differences in frequency response. In order to verify this assumption, we performed simulations for the PIN-PD, using different contact designs and antenna geometries. The results show that these parameters indeed determine the pronounced power drop around $250 \mathrm{GHz}$. Beyond $400 \mathrm{GHz}$, this influence becomes negligible, as the reactance of antenna and photodiode approach zero whereas the resistance of the antenna is constant. (Details of these simulations will be presented in a future paper.) Similarly, the strong decrease in the terahertz output power of the UTC-PD around $130 \mathrm{GHz}$ can be attributed to frequency-dependent impedance matching. The frequency-independent impedance predicted by Babinet's principle is only valid for antenna dimensions greater than the wavelength. Thus, there exists a low-frequency cutoff for real bow-tie antennas. In case of the UTC emitter, the decrease of radiated power around $70 \mathrm{GHz}$ and $110 \mathrm{GHz}$ is attributed to the frequency dependence of the antenna impedance. Additionally, the dimensions of the antenna of the UTC-PD are designed for efficient $\mathrm{THz}$ emission beyond $300 \mathrm{GHz}$ only.

One would expect that the transit-time limit of the charge carriers gives rise to another low pass with bandwidth $f_{t}=3.5 \bar{v} /\left(2 \pi d_{\text {abs }}\right)$. Here, $\bar{v}$ is the average carrier drift velocity and $d_{\mathrm{abs}}$ is the thickness of the absorber that the carriers have to traverse. However, the power spectra of 
Fig. 4 do not show any evidence of a transit-time contribution. Thus, the influence of the transit-time still remains to be investigated. Since the absorber thickness $d_{\mathrm{abs}}$ determines not only the carrier transit-time but also the capacitance of the PD and the volume of the light absorbing area, this parameter has to be designed very carefully. Note that in the UTC-PD, only the drift velocity of electrons contributes to the transit-time, which reduces the design restrictions for the absorber layer.

Another significant difference between PIN- and UTC-PDs is the DC photocurrent of the two devices. Under the conditions given in Fig. 4, the photocurrent of the PIN-PD amounts to $8.9 \mathrm{~mA}$, whereas the UTC-PD produces no more than $4.6 \mathrm{~mA}$. Since a poor optical coupling efficiency and a thin absorber are the price to pay for the UTC design, the UTC-PD shows only half of the responsivity of the PIN-PD. However, due to the advantageous carrier dynamics, the UTC-PD still achieves a comparable terahertz output power.

Next, we acquired detailed parameter maps of the terahertz output of both emitters as a function of optical power and bias voltage. Figure 6 shows contour plots of the measured terahertz power at $100 \mathrm{GHz}, 300 \mathrm{GHz}, 500 \mathrm{GHz}$, and $700 \mathrm{GHz}$. The power is normalized to the maximum of each plot and the colors represent a logarithmic scale. The normalization allows investigating the stability of the terahertz emission under varying operating conditions. Graphs in the left and right column in Fig. 6 depict the results obtained with the UTC-PD and PIN-PD, respectively. In each subplot, dark red areas represent the maximum terahertz power emitted by the device and dark blue colors correspond to the noise floor of the detector, which is reached at power levels around $1 \mu \mathrm{W}$. The contour lines enclose areas in which the emitted terahertz power remains $-1 \mathrm{~dB},-3 \mathrm{~dB},-6 \mathrm{~dB}$, and $-9 \mathrm{~dB}$ below the respective maximum. The graphs illustrate that both types of emitters require more than $30 \mathrm{~mW}$ of optical power to reach their maximum output. In addition, with both emitters, the maximum power shifts towards lower bias amplitudes with increasing frequency. Up to $500 \mathrm{GHz}$, the PIN-PD covers larger $1-\mathrm{dB}$ and $3-\mathrm{dB}$ areas than the UTC-PD. This may be an advantage to ensure stable operation under varying operating conditions, e.g., due to environmental influences in a nonlaboratory setting. In a $300-\mathrm{GHz}$ communication link, for example, the optical power at the terahertz emitter can vary depending on the modulation format.

Note that at all frequencies, the UTC-PD reaches its highest output power at $50 \mathrm{~mW}$ illumination. In contrast, the PIN-PD performs best between 35 and $40 \mathrm{~mW}$. Higher optical powers cause a roll-over behavior of the terahertz output, which points towards a screening effect: the intense optical power produces a high density of positive and negative charge carriers, which then attenuate the external electric field. On the opposite, the UTC-PD does not show any rollover or screening effect, which is explained by the lack of holes in the active region.

The power maps of Fig. 6 allow us to revisit the operating conditions for both devices. Hence, we attempted to identify a parameter set that maximizes the terahertz output in particular at high frequencies, i.e., $700 \mathrm{GHz}$ to $1 \mathrm{THz}$. For the UTC-PD, we deduce from Fig. 6 that the terahertz power increases with both bias voltage and optical input, and the "recommended" conditions of Fig. 4 ( $-1.0 \mathrm{~V}$ device bias and $30 \mathrm{~mW}$ optical feed) are not yet the optimum ones. For the PIN-PD, we find that an optical power higher than $30 \mathrm{~mW}$ is beneficial for achieving a high terahertz output, while a bias voltage of $-1.5 \mathrm{~V}$ seems adequate.

In Fig. 7, we show power spectra obtained with optimized parameter sets. The upper panel represents the power increase in $\mathrm{dB}$ compared with the recommended operating conditions of Fig. 4, and the lower panel depicts spectra of the absolute power. Note that the optimized parameters increase the output of both emitters at all frequencies. In the low-frequency range $(<100 \mathrm{GHz})$, the PIN-PD even exceeds $0 \mathrm{dBm}$. Above $500 \mathrm{GHz}$, the new parameter set 


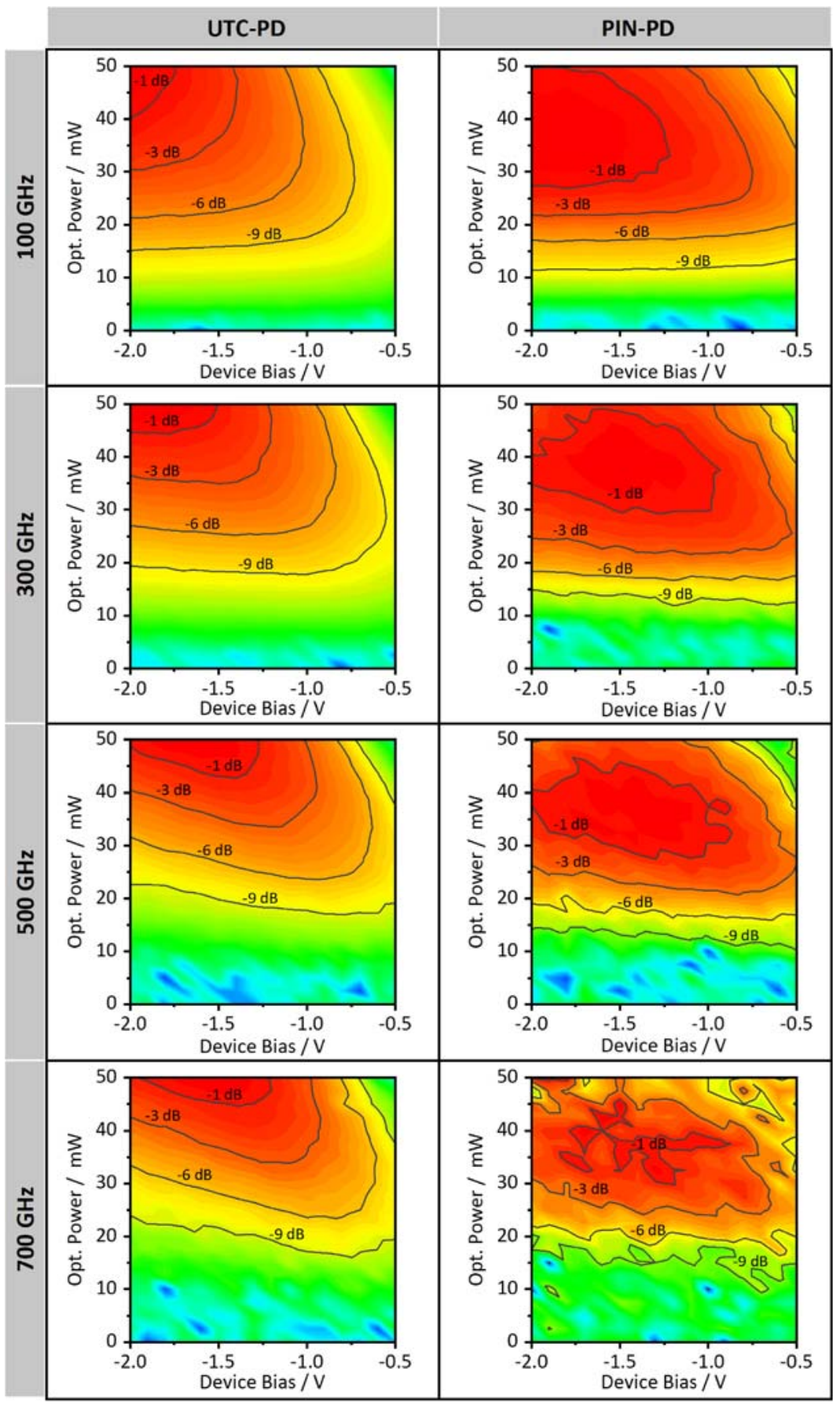

Fig. 6 Emitted terahertz power of the UTC-PD (left column) and PIN-PD (right column) modules as a function of optical power ( 0 to $50 \mathrm{~mW}$, vertical axis) and device bias ( -2.0 to $-0.5 \mathrm{~V}$, horizontal axis) for $100 \mathrm{GHz}, 300 \mathrm{GHz}$, $500 \mathrm{GHz}$, and $700 \mathrm{GHz}$. In each of the contour graphs, the power is normalized to the maximum and plotted on a logarithmic scale. The lines indicate parameter ranges that correspond to a power drop of $1 \mathrm{~dB}, 3 \mathrm{~dB}, 6 \mathrm{~dB}$, and $9 \mathrm{~dB}$ 


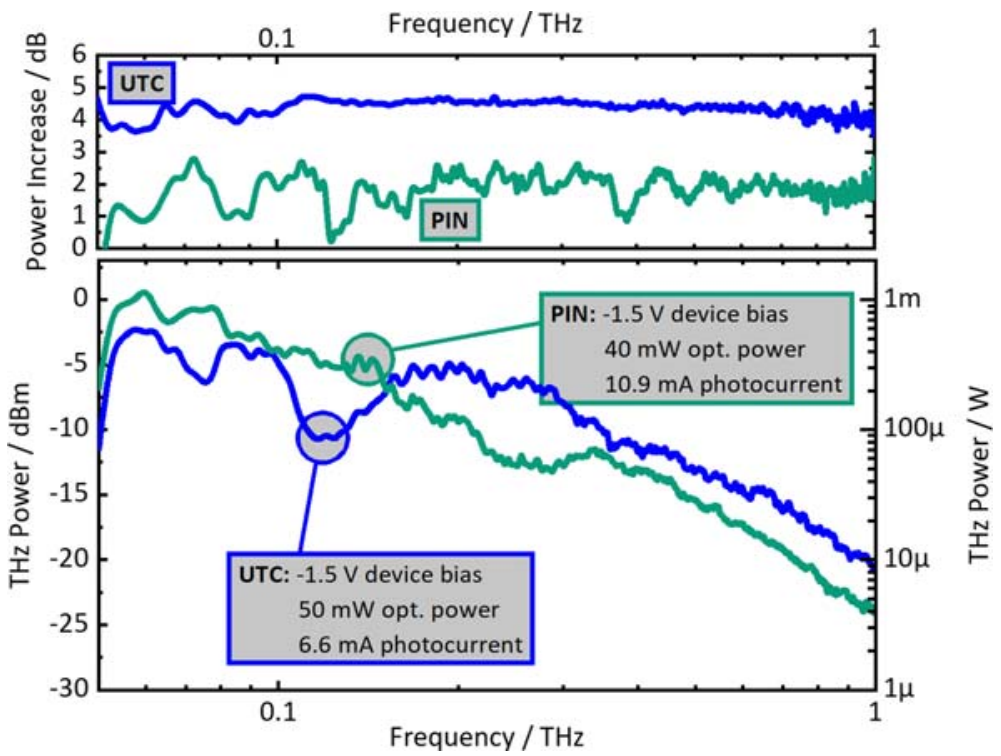

Fig. 7 Absolute value (lower plot) of the emitted terahertz power between $50 \mathrm{GHz}$ and $1 \mathrm{THz}$ for the UTC-PD and PIN-PD at optimized operation parameters derived from the power maps at $700 \mathrm{GHz}$. $-1.5 \mathrm{~V}$ device bias and $50 \mathrm{~mW}$ optical power for the UTC-PD, and $-1.5 \mathrm{~V}$ device bias and $40 \mathrm{~mW}$ optical power for the PIN-PD. The increase in terahertz output compared with recommended parameters is shown in the upper panel

increases the terahertz output by $4.3 \mathrm{~dB}$ and $2.8 \mathrm{~dB}$ on average for the UTC-PD and PIN-PD, respectively. For instance, at $1 \mathrm{THz}$, the UTC-PD reaches an output power of $-20.9 \mathrm{dBm}$, and the terahertz power emitted by the PIN-PD is only $3 \mathrm{~dB}$ lower. Note that the increase in terahertz power is almost spectrally flat for both devices. This means that the spectra of Fig. 4 appear more or less offset-shifted to higher levels in Fig. 7. While we are aware that increasing the bias voltage and optical power beyond the limits given by the manufacturers might compromise the lifetime of the devices, a long-term study is beyond the scope of this work.

\section{Conclusion}

To the best of our knowledge, we performed the first experimental comparison of a UTC-PD and a PIN-PD for continuous-wave terahertz emission between $50 \mathrm{GHz}$ and $1 \mathrm{THz}$. We measured the absolute power with a calibrated pyroelectric detector and quantified the contributions of optical input power and bias voltage. We found that when the UTC-PD and the PIN-PD are operated as recommended by the respective manufacturer, they produce comparable terahertz output spectra. However, at frequencies lower than $400 \mathrm{GHz}$, the electrical properties of the terahertz antenna and the contact design of the emitter result in prominent features in the power spectrum. At higher frequencies, a first-order low pass dominates the spectral output of both devices, which leads to a 20-dB decrease per decade. We attribute this behavior to the RC limitation, i.e., the resistance and capacitance of the photodiodes. We further measured the emitted terahertz power as a function of optical input power and bias voltage. We found that charge carrier screening leads to a roll-over behavior of the terahertz output at optical powers higher than $40 \mathrm{~mW}$ in the PIN-PD. The UTC-PD, on the 
other hand, does not reveal any saturation effects up to $50 \mathrm{~mW}$ optical input. With optimized driving parameters, the output power for frequencies beyond $500 \mathrm{GHz}$ could be increased by $4.3 \mathrm{~dB}$ and $2.8 \mathrm{~dB}$ for the UTC-PD and PIN-PD, respectively, as compared with the recommended operating conditions. A possible impact on the reliability of the devices due to altered operating condition still remains to be investigated.

In addition, we characterized four common materials for infrared (IR) absorption. Since residual $1.5 \mu \mathrm{m}$ laser light can distort any terahertz power measurements, accurate IR suppression is mandatory. Ceramic $\left(\mathrm{Al}_{2} \mathrm{O}_{3}\right)$ and cardboard absorbers turned out to block IR light very well; however, they also absorbed the terahertz radiation, especially at frequencies higher than $600 \mathrm{GHz}$. Foamed polystyrene revealed a spectrally flat terahertz transmission of more than $90 \%$ while blocking more than $98 \%$ of the residual IR. However, polystyrene consists of millimeter-sized grains, which lead to a variation in the optical attenuation when the absorber is moved. The best compromise for high IR blocking, high terahertz transmission, and good homogeneity was found with a $20 \mu \mathrm{m}$ thick piece of HDPE foil. We therefore chose this IR filter for our terahertz power measurements.

Open Access This article is distributed under the terms of the Creative Commons Attribution 4.0 International License (http://creativecommons.org/licenses/by/4.0/), which permits unrestricted use, distribution, and reproduction in any medium, provided you give appropriate credit to the original author(s) and the source, provide a link to the Creative Commons license, and indicate if changes were made.

\section{References}

1. M. Yahyapour et al., "Fastest thickness measurements with a terahertz time-domain system based on electronically controlled optical sampling," Appl. Sci., vol. 9, no. 7, p. 1283, 2019.

2. I. S. Gregory, R. K. May, K. Su, and J. A. Zeitler, "Terahertz car paint thickness sensor: out of the lab and into the factory," in International Conference on Infrared, Millimeter, and Terahertz Waves, IRMMW-THz, 2014.

3. S. Brinkmann, N. Vieweg, G. Gärtner, P. Plew, and A. Deninger, "Towards quality control in pharmaceutical packaging : screening folded boxes for package inserts," J. Infrared Millim. Terahz Waves, vol. 38, no. 3, pp. 339-346, 2017.

4. D. M. Mittleman, "Twenty years of terahertz imaging [invited]," Opt. Express, vol. 26, no. 8, pp. 94179431, 2018.

5. K. Kawase, Y. Ogawa, Y. Watanabe, and H. Inoue, "Non-destructive terahertz imaging of illicit drugs using spectral fingerprints," Opt. Express, vol. 11, no. 20, pp. 2549-2554, Oct. 2003.

6. S. Zhong et al., "Non-destructive quantification of pharmaceutical tablet coatings using terahertz pulsed imaging and optical coherence tomography," Opt. Lasers Engeneering, vol. 49, no. 3, pp. 361-365, 2011.

7. E. Pickwell and V. P. Wallace, "Biomedical applications of terahertz technology," J. Phys. D. Appl. Phys., vol. 39, no. 17, pp. R301-R310, Sep. 2006.

8. T. Nagatsuma, G. Ducournau, and C. C. Renaud, "Advances in terahertz communications accelerated by photonics," Nat. Photonics, vol. 10, no. 6, pp. 371-379, 2016.

9. G. Ducournau et al., "THz communications using photonics and electronic devices: the race to data-rate," J. Infrared, Millimeter, Terahertz Waves, vol. 36, no. 2, pp. 198-220, 2015.

10. S. Preu, G. H. Döhler, S. Malzer, L. J. Wang, and A. C. Gossard, "Tunable, continuous-wave terahertz photomixer sources and applications," J. Appl. Phys., vol. 109, no. 061301, 2011.

11. T. Göbel, D. Stanze, B. Globisch, R. J. B. Dietz, H. Roehle, and M. Schell, "Telecom technology based continuous wave terahertz photomixing system with 105 decibel signal-to-noise ratio and 3.5 terahertz bandwidth.," Opt. Lett., vol. 38, no. 20, pp. 4197-4199, Oct. 2013.

12. S. Nellen, B. Globisch, A. P. Anthur, V. Vujicic, L. Barry, and M. Schell, "Fiber-coupled, photoconductive receiver for heterodyne detection up to $1 \mathrm{THz}$ stabilized by an optical frequency comb," in International Conference on Infrared, Millimeter, and Terahertz Waves, IRMMW-THz, 2017.

13. L. Liebermeister, S. Nellen, R. Kohlhaas, S. Breuer, M. Schell, and B. Globisch, "Ultra-fast, highbandwidth coherent cw THz spectrometer for non-destructive testing," J. Infrared, Millimeter, Terahertz. Waves, vol. 40, no. 3, pp. 288-296, 2019. 
14. D. Stanze, A. Deninger, A. Roggenbuck, S. Schindler, M. Schlak, and B. Sartorius, "Compact cw terahertz spectrometer pumped at $1.5 \mu \mathrm{m}$ wavelength," J. Infrared, Millimeter, Terahertz Waves, vol. 32, no. 2, pp. 225-232, 2011.

15. J. E. Bowers and C. A. Burrus, "Ultrawide-band long-wavelength p-i-n photodetectors," J. Light. Technol., vol. 5, no. 10, pp. 1339-1350, 1987.

16. T. Ishibashi, N. Shimizu, S. Kodama, H. Ito, T. Nagatsuma, and T. Furuta, "Uni-traveling-carrier photodiodes," in Ultrafast Electronics and Optoelectronics, 1997, vol. 13, pp. 83-87.

17. H. Ito, S. Kodama, Y. Muramoto, T. Furuta, T. Nagatsuma, and T. Ishibashi, "High-speed and high-output InP-InGaAs unitraveling-carrier photodiodes," IEEE J. Sel. Top. Quantum Electron., vol. 10, no. 4, pp. 709-727, Jul. 2004.

18. M. Natrella et al., "Accurate equivalent circuit model for millimetre-wave UTC photodiodes," Opt. Express, vol. 24, no. 5, pp. 4698-4713, Feb. 2016.

19. H.-G. Bach, A. Umbach, S. van Waasen, R. M. Bertenburg, and G. Unterbörsch, "Ultrafast monolithically integrated InP-based photoreceiver: OEIC-design, fabrication, and system application,” IEEE J. Sel. Top. Quantum Electron., vol. 2, no. 2, pp. 418-423, 1996.

20. D. Stanze et al., "Coherent cw terahertz system employing photodiode emitters," in International Conference on Infrared, Millimeter, and Terahertz Waves, IRMMW-THz, 2009.

21. T. Ishibashi, Y. Muramoto, T. Yoshimatsu, and H. Ito, "Unitraveling-carrier photodiodes for terahertz applications,” IEEE J. Sel. Top. Quantum Electron., vol. 20, no. 6, pp. 79-88, Nov. 2014.

22. F. Nakajima, T. Furuta, and H. Ito, "High-power continuous-terahertz-wave generation using resonantantenna-integrated uni-travelling-carrier photodiode," Electron. Lett., vol. 40, no. 20, 2004.

23. C. C. Renaud, M. Natrella, C. Graham, J. Seddon, F. Van Dijk, and A. J. Seeds, "Antenna integrated THz uni-traveling carrier photodiodes," IEEE J. Sel. Top. Quantum Electron., vol. 24, no. 2, 2018.

24. S. Hisatake, J. Kim, and K. Ajito, "Self-Heterodyne Spectrometer Using for Terahertz-Wave Generators and Optoelectronic Mixers," vol. 32, no. 20, pp. 3683-3689, 2014.

25. E. Rouvalis, M. J. Fice, C. C. Renaud, and A. J. Seeds, "Optoelectronic detection of millimetre-wave signals with travelling-wave uni-travelling carrier photodiodes," Opt. Express, vol. 19, no. 3, pp. 2079-2084, 2011.

26. E. Rouvalis, C. C. Renaud, D. G. Moodie, M. J. Robertson, and A. J. Seeds, "Continuous wave Terahertz generation from ultra-fast InP-based photodiodes," IEEE Trans. Microw. Theory Tech., vol. 60, no. 3, pp. 509-517, 2012.

27. T. Nagatsuma, H. Ito, and T. Ishibashi, "High-power RF photodiodes and their applications," Laser Photonics Rev., vol. 3, no. 1-2, pp. 123-137, Feb. 2009.

28. A. Steiger, M. Kehrt, C. Monte, and R. Müller, "Traceable terahertz power measurement from $1 \mathrm{THz}$ to 5 THz," Opt. Express, vol. 21, no. 12, pp. 14466-14473, 2013.

29. B. Globisch et al., "Absolute terahertz power measurement of a time-domain spectroscopy system," Opt. Lett., vol. 40, no. 15, pp. 3544-3547, 2015.

30. Y. Mushiake, "Self-complementary antennas," IEEE Antennas Propag. Mag., vol. 34, no. 6, pp. 23-29, Dec. 1992.

31. V. H. Rumsey, "Frequency independent antennas," in IRE International Convention Record, 1966, vol. 5, pp. 114-118.

Publisher's Note Springer Nature remains neutral with regard to jurisdictional claims in published maps and institutional affiliations. 